\title{
Indeterminate Growth: Could It Represent the Ancestral Condition?
}

\author{
Iswar K. Hariharan ${ }^{1}$, David B. Wake², and Marvalee H. Wake \\ ${ }^{1}$ Department of Molecular and Cell Biology, University of California, Berkeley, California 94720 \\ ${ }^{2}$ Department of Integrative Biology, University of California, Berkeley, California 94720 \\ Correspondence: ikh@berkeley.edu; wakelab@berkeley.edu; mhwake@berkeley.edu
}

\begin{abstract}
Although we are used to the idea that many organisms stop growing when they reach a predictable size, in many taxa, growth occurs throughout the life of an organism, a phenomenon referred to as indeterminate growth. Our comparative analysis suggests that indeterminate growth may indeed represent the ancestral condition, whereas the permanent arrest of growth may be a more derived state. Consistent with this idea, in diverse taxa, the basal branches show indeterminate growth, whereas more derived branches arrest their growth. Importantly, in some closely related taxa, the termination of growth has evolved in mechanistically distinct ways. Also, even within a single organism, different organs can differ with respect to whether they terminate their growth or not. Finally, the study of tooth development indicates that, even at the level of a single tissue, multiple determinate patterns of growth can evolve from an ancestral one that is indeterminate.
\end{abstract}

W hen we think of adult mammals, especially humans, we think of growth having ceased. That is, there is no further increase in the length of the major body axis because the long bones and vertebrae no longer grow. In fact, slow shrinkage becomes the norm, although we also are aware that some tissues, notably adipose tissue, and in humans, ears and noses, continue to grow; enormous weight gains can occur in adults. Thus, we are familiar with organisms that achieve a predictable size and form at the end of their development. The ways that the growth and proliferation of individual cells is arrested as an organism approaches its final size and shape are currently the subject of intense investigations. However, many organisms, from diverse kingdoms and phyla, show the property of indeterminate growth (Sebens 1987) - a condition in which the growth of the organism likely occurs throughout its life. Mammals, among a few other taxa, typically, appear to show determinate growth, and humans are a prime example. Here, we examine the growth properties of organisms from diverse taxa in an attempt to understand whether determinate growth or indeterminate growth represents the ancestral condition.

Organisms differ greatly in size and shape, even within clades, and when comparing them, one must proceed using appropriate comparative methods and phylogenetic hypotheses. There has been a continuous unfolding of the grand tree of life, but along the way, there have been many reversals and fresh starts. Without a phylogenetic hypothesis, it is impossible to tell "which way is up." The tree of life has taken on more and more structure, and the time is right to use it to examine cell-level properties, such as

Editors: Rebecca Heald, Iswar K. Hariharan, and David B. Wake

Additional Perspectives on Size Control in Biology: From Organelles to Organisms available at www.cshperspectives.org

Copyright (C) 2016 Cold Spring Harbor Laboratory Press; all rights reserved; doi: 10.1101/cshperspect.a019174

Cite this article as Cold Spring Harb Perspect Biol 2016;8:a019174 
I.K. Hariharan et al.

growth, development, and regeneration; there is a rapidly growing literature. Here, we examine similarities and differences between those organisms that clearly stop growing and closely related organisms that do not.

\section{ONTOGENETIC TRAJECTORY: A FRAMEWORK FOR STUDYING WHEN AND HOW GROWTH CEASES}

To move beyond a discussion of growth that is purely descriptive and to emphasize the development of an organism as a dynamic process, the concept of an ontogenetic trajectory was developed. A detailed and more mathematical treatment of the subject is covered by Alberch et al. (1979) and Alberch (1980). Here, we present the essence of the concept in a highly simplified way and emphasize its utility in comparing the growth properties of different organisms both within and among species.

The ontogenetic trajectory of an organ or organism is a mathematical representation of the pathway that it follows from the beginning of its development through to its mature form. This is an idealization that is no less than the complete record of the physical appearance of a system through time. The antecedents of this idealization are ancient, going back to one of the founders of developmental biology, Karl von Baer, and including the early evolutionist Ernst Haeckel, who argued that evolution was the modification of ontogeny and was perhaps the first to place evolutionary changes in ontogeny into the context of a phylogenetic tree.

Ontogenetic trajectories can be realized by selecting an arbitrary starting point in the development of an organ or organism, measuring it, and then making comparable measurements as growth occurs. Alternatively, development of an organ, for example, a skull, can be studied by recording the time of appearance of its constituent bones. For simplicity, the progress of an organ or organism can be envisioned as a point moving through three dimensions: size, shape, and age (Fig. 1A). "Shape" refers to a parameter that could be defined as the ratio of multiple measurements that together convey a sense of changes in form. Unless growth is strictly isometric (rarely encountered), "shape" changes with respect to the age of the organism (allometry) (Huxley 1932; Gould 1977). A three-dimensional plot showing a hypothetical ontogenetic trajectory is shown in Figure 1A. Different individuals of the same species and gender have similar ontogenetic trajectories. Thus, a population can be visualized as a "cloud" of points tracing closely similar ontogenetic trajectories. Standard statistics can then be applied and a mean ontogenetic trajectory determined. This allows a quantitative comparison of the ontogeny of different species.

Most of the ontogenetic trajectories can be linearized and the trajectory for a species that is used as reference can be represented as a straight line with slope $k$, with a characteristic onset and offset (Fig. 1B). This, of course, does not mean that the organism increases in size at a linear rate; this representation is merely convenient from a mathematical viewpoint. A related species may have a different mean trajectory, which can be compared and analyzed with respect to factors responsible for differences. For example, if one takes the ontogenetic trajectory of species 1 as a starting point, that of species 2 , which reaches a larger size and, hence, a different shape, could occur either because the species has a higher rate of growth, an earlier onset of growth, or a delayed offset signal.

In each of these idealized examples, the species arrests its increase in size at a specific point in size-shape-age space. However, in reality, most organisms seldom maintain a constancy of size and shape throughout their lives. For those organisms that show determinate growth, a maximal size is attained at some point in development. For those that show indeterminate growth, it is not obvious that the growth process has ceased at the end of its life span. Thus, the ontogenetic trajectory of the organism would continue along a path of increasing size (Fig. 1C), although its rate of growth could keep decreasing. What makes the phenomenon of indeterminate growth difficult to define with complete certainty is the possibility that some organisms may eventually stop growing at an age that far exceeds their life span under normal 
A

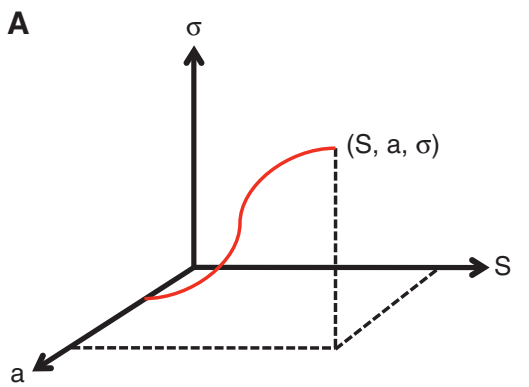

B

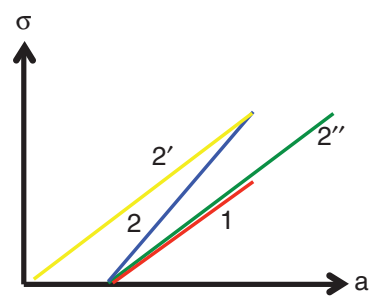

C

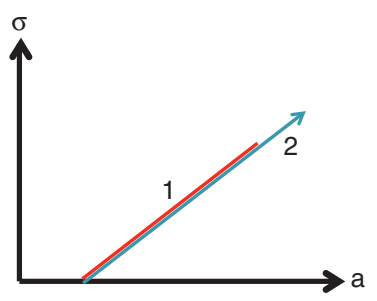

Figure 1. Ontogenetic trajectories. $(A)$ The ontogenetic trajectory of an organism is shown as a point moving in three dimensions: size $(S)$, shape $(\sigma)$, and age $(\mathrm{a}) .(B)$ Linearized ontogenetic trajectories are shown as a plot of shape $(\sigma)$ versus age (a). Species 2 can achieve a final shape that is different to that of species 1 either by having a different rate of shape change (2), a different time of onset of growth $\left(2^{\prime}\right)$, or a delayed offset $\left(2^{\prime \prime}\right)$. (C) Species 1 has determinate growth, whereas species 2 has indeterminate growth. By showing plots of shape versus age, rather than size versus age, the growth characteristics of species that differ considerably in size can be easily compared.

conditions. With this caveat in mind, we will apply the term indeterminate growth to those organisms for which there is no clear evidence for a developmental mechanism that arrests growth. First, we consider several invertebrate taxa in which the study of specific model organisms has provided important insights into mechanisms that regulate growth. Second, we examine the distribution of determinate and indeterminate growth among vertebrates. Third, we discuss the development of teeth within vertebrate taxa to illustrate how the patterns of initiation and cessation of growth can differ widely even within a single tissue.

\section{GROWTH PROPERTIES OF INVERTEBRATES}

Among invertebrates, we have focused on four major taxa-arthropods, annelids, planaria, and hydrozoa, because an examination of each of these provides interesting insights into mechanisms underlying the termination or persistence of growth. These taxa belong to diverse phyla. The phylum Arthropoda belongs to the superphylum Ecdysozoa, whereas Annelida and planaria belong to the superphylum Lophotrochozoa. Lophotrochozoa include segmented worms (Annelida) as well as the phyla Mollusca and Platyhelminthes ( planarians and other flatworms, tapeworms) and many others (Ferrier 2012). Hydrozoa belong to the phylum Cnidaria.

\section{Growth Patterns in Arthropods}

Both arthropods and annelids are segmented. However, unlike annelids, where the number of segments can continue to increase throughout life, most arthopods generate a fixed number of segments relatively early in development and growth subsequently occurs by an increase in size of the segments. Because most arthropods have a rigid exoskeleton, growth occurs intermittently between molts when the old integument is shed. Thus, those that show a determinate pattern of growth have a fixed number of molts, whereas those that have indeterminate growth continue to molt indefinitely.

Among the arthropods, most studies of growth have been conducted on crustaceans and insects. Recent phylogenetic studies (Giribet and Edgecombe 2012) favor a model in which Hexapoda (which includes insects) arises as a monophyletic (including an ancestor and all of its descendants) group from within the Crustacea, thus making Crustacea a paraphyletic (including an ancestor but only some of its descendants) taxon. Although the growth properties of relatively few crustacean species have been characterized, clear differences in their growth properties emerge; some taxa have been documented to show indeterminate growth, whereas others show determinate growth (Hartnoll 1983, 2001). For example, the Branchiopoda, which are characterized by the presence of gills 
I.K. Hariharan et al.

on their appendages and include the water flea Daphnia, show indeterminate growth (Yampolsky and Ebert 1994). In contrast, the Ostracoda, whose bodies are encased in two chitinous valvelike structures, and the Copepoda terminate their growth at a specific stage and have a defined number of molts. Even in those crustaceans that have indeterminate growth, the rate of growth slows down as the organism ages, both by increasing the time between successive molts and by decreasing the extent of growth that occurs between molts (Hartnoll 1983). Thus, in practice, growth appears to level off as if the organism does have an upper size limit.

Within the order Decapoda, which includes lobsters and crabs, there is a diversity of growth patterns. The lobster Homarus has indeterminate growth, whereas within the group of crabs, growth is determinate in some species and indeterminate in others. The characteristics of growth have been studied in relatively few species of crabs, thus precluding definitive phylogenetic conclusions. Despite this, there are hints that a condition of determinate growth could have evolved from an ancestral pattern of indeterminate growth by distinct mechanisms in related species. Like lobsters, some crabs, such as Cancer magister (the Dungeness crab) and Paralithodes camtschaticus (the red king crab), show indeterminate growth and continue to molt throughout their lives (Wainwright and Armstrong 1993; Zhou et al. 1998), whereas others stop molting when they reach sexual maturity. Remarkably, in two species of crabs that show determinate growth, the shore crab Carnicus maenas and the spider crab, Acanthonyx lunulatus, the cessation of molting occurs for different reasons. Two important regulators of molting are a steroid hormone, which promotes molting, 20-hyroxyecdysone, made by the molt glands or Y-organs (Lachaise et al. 1993), and the peptide hormone molt-inhibiting hormone $(\mathrm{MIH})$, which is produced by neurosecretory cells in the eyestalk (Webster and Keller 1986). In C. maenas, there is continued production of high levels of MIH after the terminal molt that likely prevents further molting. In A. lunulatus, the Y-organs degenerate, thus preventing the production of 20-hydroxyecdysone necessary for molting (Hartnoll 2001). Thus, even among crabs, the mechanistic basis underlying the cessation of molting can differ between species, suggesting that determinate growth could have evolved in a variety of ways from indeterminate growth.

Among insects, the earliest branching taxa show indeterminate growth, whereas growth is clearly determinate in more derived taxa, such as Diptera (flies), thus supporting the conjecture that the ancestral condition may be one of indeterminate growth (Fig. 2A). Ametabolous insects, which are basal branches in the insect radiation (Giribet and Edgecombe 2012), lack wings (apterygote hexapods) and include the Archeognatha (bristletails) and the Zygentoma (silverfish). The newly hatched organism resembles the adult in most ways except that it is much smaller. Growth occurs between molts, yet the overall form of the organism remains relatively constant. The apterygote hexapods are reported to show indeterminate growth and some have been reported to undergo as many as 50 molts (Richards and Davies 1977). Their long-term growth properties have, thus far, received relatively little attention.

The hemimetabolous insects, which include cockroaches, crickets, dragonflies, and the true bugs (Hemiptera), undergo an incomplete metamorphosis. The embryo hatches into a nymph, which resembles the adult in some ways but is smaller and lacks wings or genitalia. A series of molts terminates with a final imaginal molt that results in an adult insect with functional reproductive organs and extended wings. Molting in insects is promoted by increasing levels of a steroid hormone produced by the prothoracic gland (PG). In cockroaches, the PG is broken down after the final imaginal molt, thus ensuring that no further molts can occur (Mané-Padrós et al. 2008, 2010). However, implanting multiple PGs can cause adults to molt (Bodenstein 1953), thus demonstrating that the presence of these tissues is sufficient for molting to occur. Thus, the cessation of molting and, hence, growth occurs by the destruction of the PG.

In the holometabolous insects, a clade that includes flies, moths, beetles, and wasps, the 
Indeterminate Growth

A

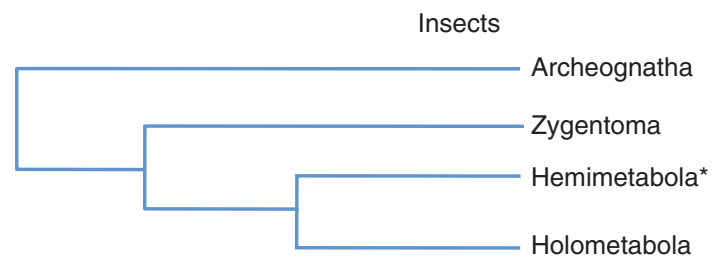

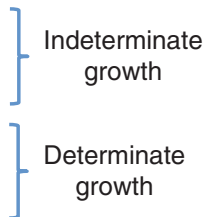

growth

\section{B}

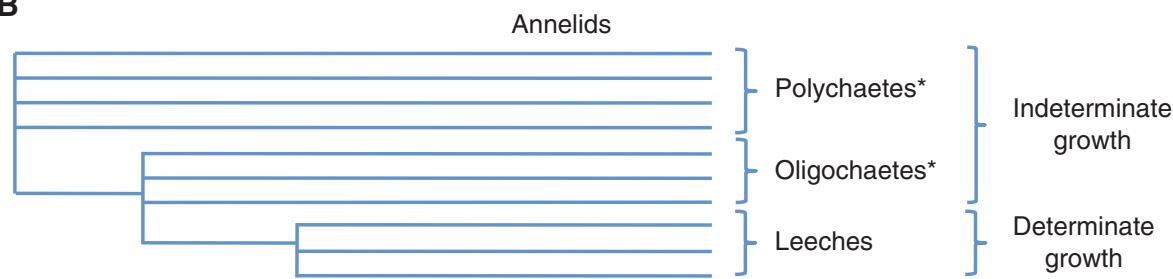

Figure 2. Phylogenetic relationships of $(A)$ insect (Giribet and Edgecombe 2012), and $(B)$ annelid (Ferrier 2012) species. Note that the more basal branches show indeterminate growth. The asterisks indicate paraphyletic taxa.

final larval instar results in pupariation, destruction of the larval body, and a complete metamorphosis when the adult body is generated from primordia within the larva known as imaginal discs. The hormonal regulation of metamorphosis has been studied most intensively in the moth Manduca sexta and the fly Drosophila melanogaster (Rewitz et al. 2013; Nijhout et al. 2014). In both organisms, the timing of the molts and of entry into metamorphosis is regulated by an interplay between juvenile hormones (JHs), which are derivatives of farnesoic acid made by paired glands, the corpora allata, and the steroid hormone ecdysone made by the PG. In Drosophila, a pulse of ecdysone precedes the first two molts. The last (third) larval instar is characterized by three ecdysone pulses of relatively low amplitude and a final surge of ecdysone production at the time of onset pupariation. The PG is destroyed together with many other larval tissues during metamorphosis thus preventing further molts from occurring (Dai and Gilbert 1991). In Drosophila, an additional mechanism that involves terminal differentiation of precursor cells, precludes further growth. Much of the adult epidermis is generated from cells of the larval imaginal discs. These cells are potentially capable of indefinite proliferation, as shown by serial transplantation of imaginal disc frag- ments (Hadorn 1968), and can, therefore, be regarded as a lineage-specific pool of progenitor cells with the potential for continued proliferation. The extrinsic controls imposed on these cells during metamorphosis alter their proliferative properties such that they complete their final divisions during the pupal stage and arrest permanently in a postmitotic state (Buttitta and Edgar 2007). The destruction of the PG together with the terminal differentiation of imaginal disc cells results in a definitive termination of both molting and growth in these insects.

\section{Annelid Growth Patterns}

A particularly illuminating case of how a state of determinate growth can derive from an ancestral species that has indeterminate growth occurs among the annelids (Fig. 2B). Annelida is a phylum of segmented worms that belongs to the superphylum Lophotrochozoa, along with the phyla Mollusca and Platyhelminthes (planarians and other flatworms, tapeworms) and many others (Ferrier 2012). Among the annelids is a large paraphylectic group, the Polychaeta, whose members have large numbers of bristles or chaetae that are used for locomotion. Nested within this group is a monophyletic group, the Clitellata, which are composed of a paraphyletic group, the Oligochaeta (which have fewer chae- 
I.K. Hariharan et al.

tae than the polychaetes) and a single branch from which derive the Hirudinea (the leeches). Most polychaete annelids generate a small number of anterior segments during embryonic and larval stages and then proceed to add segments throughout their lives (de Rosa et al. 2005). In contrast, the Clitellata have no independent larval stages and generate all their segments without an obvious temporal discontinuity. This addition of segments occurs toward the posterior end of the worm at a posterior subterminal growth zone. Although polychaetes keep adding segments throughout their lives, they typically die at a reasonably predictable size. For example, the polychaete Platynereis dumerilit dies shortly after reaching sexual maturity, which usually occurs when the worm has $\sim 80$ segments (Fischer et al. 2010; Gazave et al. 2013). However, there is no obvious evidence that $P$. dumerilii stops making segments before the time of death. Although this appears to be the ancestral pattern of growth among the annelids, sublineages of annelids have evolved different growth strategies. For example, the naidine annelids, a group of aquatic oligochaetes, reproduce asexually by undergoing fission that can break the worm into two pieces of similar length (Bely and Sikes 2010). Most remarkably, the leeches make exactly 32 segments and no more. In leeches, segments are generated by five pairs of lineage-restricted stem cells called teloblasts, which undergo asymmetric divisions to generate segment founder cells. Lineage-tracing experiments (Liu et al. 1998) showed that, once they have generated their full complement of segmental founder cells, the teloblasts fuse with the syncytial yolk cell. Thus, the elimination of this stem-cell population, which occurs as a result of cell-fusion events, prevents the formation of future segments.

\section{Growth and Degrowth in Planarians}

Although it is generally assumed that growth is a unidirectional process, there are several types of organisms that are capable, under conditions of nutritional deprivation, to reduce their size- a process sometimes referred to as "degrowth" (Abeloos 1930; Baguña 1998). Although this process has been studied most extensively in planarians (flatworms), it has also been reported to occur in other phyla including cnidarians and nemertines. The process of degrowth can occur to such an extent that the mature organism can reach a size that is less than its size at hatching. The ability of an organism to grow and shrink in response to changes in nutrient availability calls into question whether organisms even need to either achieve or approach a fixed final size during development.

Planarians can grow and reproduce either sexually or asexually. During asexual reproduction, the posterior end of the worm attaches to the substrate. The movement of the anterior end of the worm away from the point of attachment results in fission of the worm, typically at some point posterior to the pharynx. Each fragment grows and undergoes repatterning to restore a fully functional and complete body. For a given set of nutritional conditions and temperature, a particular flatworm species undergoes fission at a relatively predictable size. How the worm senses its size and activates behaviors that result in fission are not known. For asexually reproducing planaria reared under nutritionally favorable conditions, growth really never ceases; the growing mass is periodically partitioned into two or more parts and each continues to grow.

The cellular basis of degrowth can, in many ways, be seen as a simple reversal of growth because the rates of growth and degrowth are similar and occur at linear rates, $\sim 1 \mathrm{~mm}$ every 2 weeks for Schmidtea mediterranea (Oviedo et al. 2003). The change in size occurs mainly as a result of a change in cell number along the long axis of the worm (Baguña and Romero 1981). The cells of a flatworm undergo turnover as part of a homeostatic mechanism. Neoblasts are constantly undergoing proliferation and their differentiated progeny replace cells that have been eliminated. In such a system, growth and degrowth could potentially occur simply by altering the difference between the rate at which cells are being generated and the rate at which they are being replaced. It is, therefore, conceivable that the organism does not have any kind of mechanism that senses and adjusts its overall 
size with any precision; rather, its size may simply be determined by rates at which cells are being generated and eliminated.

The ability to use RNA-mediated interference to reduce gene activity in planarians (Newmark et al. 2003) provides exciting opportunities to identify the signaling mechanisms that regulate growth and degrowth. Indeed, a reduction in the activity of the insulin-like peptide in1 or the insulin receptor mimics starvation and causes degrowth even when the worms are feeding normally (Miller and Newmark 2012).

\section{Rejuvenation in Hydrozoans}

Although shrinkage in planarians can be considered, in some ways, a reversal of the normal ontogenetic trajectory of the organism, it is nevertheless restricted to the same developmental stage. In contrast, some members of the phylum Cnidaria, of the class Hydrozoa, are able to undergo a more dramatic reversal in their ontogenetic trajectory when a part of the organism's body can revert to a different type of structure that is characteristic of an earlier stage of development. In the "textbook" version of the hydrozoan life cycle, fertilization is followed by the development of a free-swimming larval stage of short duration referred to as a planula, which then attaches to a surface to form a primary polyp. Maturation of the polyp ultimately generates sexually competent medusae that have tentacles surrounding a mouth. Spawning by the medusae results in fertilization and the formation of the next generation of planulae. Among the hydrozoans, there are many deviations from this canonical life cycle with many showing highly truncated or even absent planula, polyp, or medusa stages. Moreover, in many species, the planula or polyp phase can arrest development. The shrinkage of a polyp colony and its subsequent expansion is referred to as "renovation." Strikingly, in some hyrozoans, a variety of chemical and environmental stresses can promote "reverse development" in immature medusae, when cells change their properties such that the features of a medusa are replaced by the differentiation of polyp-like structures that initiate the formation of a new polyp colony
(Müller 1913; Piraino et al. 2004; Schmich et al. 2007). At least, in principle, this cycle of growth and reverse development can occur indefinitely.

These examples show some of the variety of growth patterns as observed in invertebrate taxa and suggest that the idea of organs or organisms growing to a fixed final size may be a derived state. Such a state can be achieved via multiple mechanisms that could involve alterations in hormonal regulation or changes in the fate of proliferative cells, or a combination of both.

\section{PATTERNS OF GROWTH PROPERTIES OF VERTEBRATES}

Birds and mammals are usually considered to display determinate growth, but many mammals show some postmaturational growth. The spotted hyaena (Crocuta crocuta) is a good example; it matures sexually at $\sim 24$ mo but continues to grow until $32 \mathrm{mo}$, but then it stops (Swanson et al. 2013). Mammals have growth zones, cartilaginous areas at the ends of long bones and even at the ends of vertebral centra that separate an epiphyseal cap from the rest of the bone. When the cartilaginous areas ossify, skeletal growth ceases. Typically, growth slows dramatically or ceases when sexual maturity is achieved. Pathological exceptions, such as gigantism and dwarfism, are informative. Gigantism in humans is associated with acromegaly, which results from the production of excess growth hormone and which leads to progression along the ontogenetic trajectory. Individuals become very tall (often exceeding $2 \mathrm{~m}$ ) and have exaggerated physical features, such as brow ridges and large spaces between teeth. In contrast, proportional dwarfism is the result of cessation of progression along the human ontogenetic trajectory. Birds are a prime example of determinate growth. Growth in birds is under the control of plasma growth hormone, which achieves high levels following hatching and then declines (McNabb et al. 1998).

In contrast to birds and mammals, most other vertebrate taxa, as far as we can tell, show indeterminate growth. Large species of 
I.K. Hariharan et al.

fishes, both cartilaginous (e.g., sharks, rays, and chimaeras) and bony fishes grow throughout life, but the rate varies greatly among taxa. Tunas and sharks and their relatives can achieve gigantic size, and yet they continue to grow, in contrast to the enormous whales (among mammals) and ostriches (among birds). With truly miniaturized fishes, it can be difficult to document whether they show indeterminate growth or not, because of great differences among taxa in longevity-some species simply die before they achieve their growth potential. A few small teleosts might show determinate growth. But determinate growth is a derived and unusual condition for fishes, if it occurs at all. Amphibians, too, show mainly indeterminate growth (see below), although some frogs appear to cease growth before the end of life. In amniotes (reptiles, birds, and mammals), indeterminate growth is the ancestral condition. Lizards and alligators have epiphyseal growth and growth slows greatly when the epiphyses fuse to the long bones. Turtles grow throughout life, but they slow down greatly with increasing age. Tortoises, for example, continue to grow as they achieve great age (at least 180 years and possibly much older). Some miniature lizards and snakes may show determinate growth but, as far as we know, all show postmaturational growth (Shine and Charnov 1992).

Cartilage is a tissue that is especially effective for rapid and continuing growth. Even in humans, ears and noses, both with abundant cartilage, grow throughout life, but at low rates. Ossification of cartilaginous growth zones stops growth in mammals and birds, and variants of the situation can be seen in some other taxa. Miniaturization has evolved repeatedly in frogs and salamanders. The phenomenon has been best studied in salamanders, in particular in the plethodontid genus Thorius, which achieves something close to determinate growth by the early mineralization of the cartilaginous ends of bones, the intervertebral cartilages, and cartilaginous elements of the hands and feet (Hanken 1982). In miniaturized frogs of the genus Brachycephalus, sexual maturation is accompanied by hyperossification that joins the vertebrae to each other and to the skull, and by ossification of the ends of long bones and hand and foot skeletons.

Salamanders, which retain cartilaginous growth zones throughout life, can achieve gigantic size (2.8 $\mathrm{m}$ in the genus Andrias). Many species continue to add tail vertebrae throughout life, even when growth of long bones has virtually ceased. In contrast, numbers of tail vertebrae in the plethodontid salamander $\mathrm{Hy}$ dromantes cease increasing in numbers as the organism begins using its tail as a "fifth leg" during locomotion and the terminal caudal vertebra becomes morphologically specialized ("blunted").

Shrinkage during ontogeny occurs in many tissues, including portions of the brain responsible for bird song, which increase and decrease seasonally, gut length and elaboration in frogs, snakes and other organisms, which increase and decrease in response to food intake (growth can be very rapid), and, of course, adipose tissues. However, decreases in skeletal portions are poorly documented. Marine iguanas were reported to shrink prominently with low availability of food, and bone resorption was hypothesized (Wikelski and Thom 2000). Shrews are known to shrink during winter hibernation and even the braincase is said to flatten, and, although ontogeny has been studied histologically, the mechanistic and genetic controls are not yet understood (Pucek 1957). The most famous example of shrinkage in vertebrates is found in members of the South American frog genus Pseudis. Total length of the gigantic tadpoles can be three to four times that of adults. However, tadpoles typically have almost no bone. Furthermore, if one measures snout to vent length, there is virtually no shrinkage throughout metamorphosis (from developmental stage IV to stage XIII [Fabrezi et al. 2009]).

Familiar salamanders have larval and adult stages, separated by a metamorphic event. The ontogenetic trajectory of an entire organism can be envisioned as starting with a zygote that proceeds along a developmental trajectory to a first plateau, the functioning larva. The plateau represents a slowing of the progression along the developmental trajectory. After some time, progression along the trajectory dramatically in- 
creases as metamorphosis occurs, then progress slows again as the organism grows, eventually reaching a second plateau, adulthood, determined by the attainment of sexual maturity, followed by senescence.

An axolotl has the form of a very large larval salamander that is sexually mature. Its ontogenetic trajectory stalls on the shape axis, although progression continues along the size and age axes. This is the result of a change in the rate of growth from that typical of related taxa; the formal term for such a condition is neoteny. Neoteny is one of a number of phenomena known collectively as paedomorphosis, the resemblance of an adult of a descendant taxon to an earlier place on the ontogenetic trajectory of an ancestral form (Gould 1977; Alberch et al. 1979).

Many species of salamanders and other amphibians, and all amniotes, lack aquatic larval stages. Their ontogenetic trajectories reach only one plateau, adulthood. Nevertheless, parts of the organism can display neoteny. As an example, the vertebrate skull consists in part of bones that appear at different times as the skull progresses on its ontogenetic trajectory. Some bones appear early, others later, in a sequence typical of each species. Imagine that species 1 has an ossification sequence in which the prefrontal bone is the last to make an appearance. The rate of progression along the ontogenetic trajectory is slowed in species 2 , with the result that adulthood is achieved before the ossification sequence is finished and the species lacks a prefrontal. This is another example of a paedomorphic change, in this case resulting from neoteny. In other instances of heterochrony, the order of appearance of elements of the ontogeny, for example, bones of the skull, might be shuffled.

A factor directly related to evolution of ontogenetic trajectories is cell size, which is directly correlated with genome size and cell-cycle time (see the literature). Salamanders have very large genomes and, hence, have very large cells. These cells take a relatively very long time to divide. The outcome is slow progression along ontogenetic trajectories. Thus, if one thinks in terms of effective time rather than sidereal time, perhaps salamanders never achieve their full de- velopmental potential. The fact that many salamanders are small as adults means that their individual organs are made up of relatively few large cells. This, in turn, leads to a high incidence of neoteny, because of a general slowing of ontogeny.

The point of these examples is that differences in the rates of growth are strongly influenced in ectothermic vertebrates by genome size, with large-genomed species growing more slowly to much more slowly than small-genomed species. However, growth is typically indeterminate, and some neotenic species achieve enormous body sizes without ever attaining their theoretically achievable size (and shape). Thus, large-bodied species with large genomes continue to grow even though, if one normalizes by numbers of cells, one can make an argument that they are biologically smaller than species of smaller metric size (Hanken and Wake 1993).

From the above considerations, we conclude that within vertebrates determinate growth is relatively uncommon, except in birds and mammals.

Similarities between Organismal Growth in Invertebrates and the Growth of Specific Organs in Vertebrates

Although the ability to degrow is not a characteristic of most vertebrates, there are, indeed, specific vertebrate tissues whose growth properties resemble those in planarians in that terminally differentiated cells appear to be generated continuously from a population of stem cells. Interestingly, recent studies have noted that, unlike for many other organs, inactivation of Yap/ Yki, the growth-promoting transcriptional coactivator, in either the mouse intestine or in planarians results in an increase in the number of stem cells (Barry et al. 2013; Lin and Pearson 2014). The ability to shrink is also observed in the vertebrate intestine, most remarkably in those of snakes, such as the python, which intermittently feed on whole animals often months apart (Secor and Diamond 1997). Both the thickness and cellularity of the intestinal epithelium decrease markedly between meals. 
I.K. Hariharan et al.

Additionally, liver size can change in response to a variety of environmental factors. Indeed, both the intestine and liver seem especially sensitive to the levels of activity of the Hippo signaling pathway (Camargo et al. 2007; Dong et al. 2007).

\section{TEETH}

We have discussed how the evolution of a state of determinate growth from one of indeterminate growth could occur independently in individual organs resulting in a situation in which some organs in a given organism show determinate growth and others persist with indeterminate growth. Rather than studying growth in entire organisms, a more tractable problem might be the study of how growth patterns of a single tissue differ in related species with respect to the arrest of growth. The initiation and replacement of teeth in vertebrate lineages is an attractive system because a tooth derives from a relatively simple tissue; the relevant stem cells have been identified and some of the molecular regulators have been characterized.

Teeth in many clades of vertebrates (Fig. 3), especially those that branched early, are uniform in shape, polystichous (in multiple rows), first occur anteriorly then develop progressively posteriorly on the jaws, and are replaced in waves throughout life (polyphyodonty) with teeth being shed and new teeth developing from adjacent loci to replace them (e.g., most fishes, amphibians, reptiles); this is the likely ancestral condition for vertebrates. In mammals, teeth typically are of two or more specific shapes (usually correlated with function), in single rows (monostichous), and of only two sets (diphyodont), a juvenile dentation that is replaced by an adult form. The shape of the tooth crown can change as teeth are replaced during the animal's ontogeny but, in many taxa, shape is invariant. Teeth may be present in sites other than jaws, such as the pharyngeal teeth of several teleost lineages and the vomeropalatine tooth patches of some salamanders. Teeth are not replaced in some mammals; rather, as in elephants, individual teeth (incisors or molars, depending on the clade) grow throughout life, and only feeding mechanics and/or genetics keep them from over-growing in mouths. Members of yet other clades may fail to develop any teeth (e.g., birds, some frogs), or only a few specific and specialized teeth, although the developmental-genetic mechanisms may persist but remain unactivated (e.g., some fishes, some frogs, some mammals). Teleost fishes, lizards, and mammals include taxa that display several of these phenomena, even at the level of higher taxa-e.g., cichlid fishes that have polystichous teeth (whose shape can be modified by diet) on the jaws and pharyngeal elements. These evolutionarily derived variations have arisen multiple times and occur in diverse, often distantly related lineages, thus illustrating the evolutionary plasticity of the growth program of even a single, relatively simple structure.

Recently, extensive but highly topical research programs on the molecular genetics of tooth development, growth, and replacement have investigated the mechanistic basis for developmental patterns that are variously openended (continuous development and replacement) or constrained (e.g., complex teeth but replaced only once, or a single, ever-growing set). A phylogenetic/evolutionary perspective is important to understanding patterns of growth and their modifications; research on anamniotes (fishes and amphibians) is informing that on amniotes (reptiles, birds, mammals) and vice versa. Furthermore, research on nonmodel organisms is proving essential, because certain "model animals" are proving to be exceptions within their clades (e.g., Xenopus, "chick," Mus) so that information is difficult to generalize. However, major questions about growth and regeneration are potentially amenable to some resolution through research on the mechanics (molecular, genetic, and evolutionary) of development, growth, and replacement of teeth.

Mechanisms of Tooth Growth, Replacement, and Evolution

Why are mammalian ancestors, and some extinct mammals, polyphyodont, but modern mammals diphyodont? Why did zebrafish and 
Indeterminate Growth

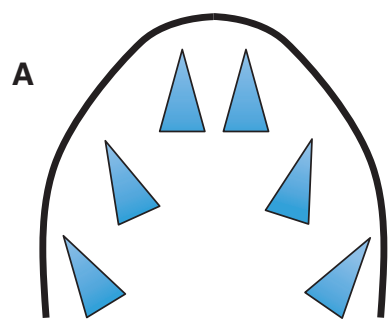

Homodonty

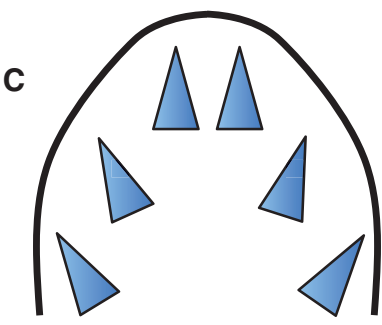

Monodonty

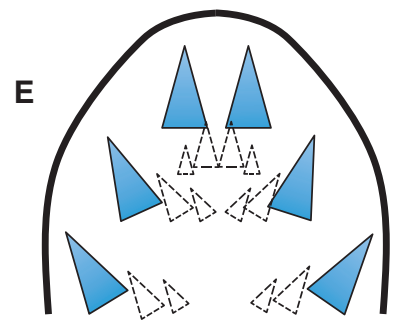

Polyphyodonty
B

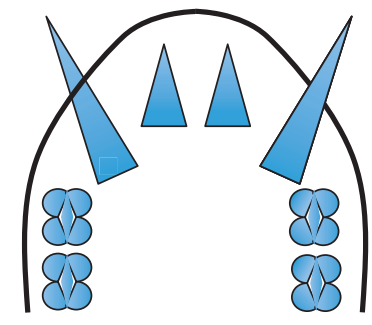

Heterodonty

D

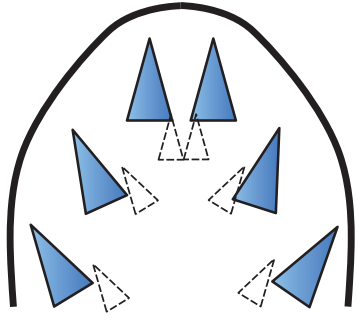

Diphyodonty

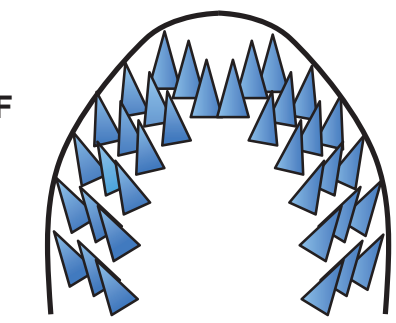

Polystichy

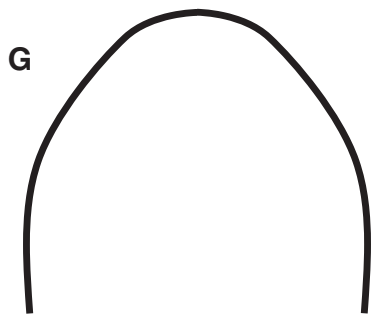

Edentulous

Figure 3. Common patterns of teeth in vertebrates. Teeth in vertebrates are variable within clades in shapes, patterns of arrangement on the jaws (or other elements), and numbers of both teeth and the rows in which they occur in adults. (A) Homodonty: all teeth in a row the same shape (many fishes, amphibians, reptiles). (B) Heterodonty: teeth different shapes in a row (e.g., the incisors, canines, and molars of many mammals). $(C)$ Monodonty: a single row of functional teeth. $(D)$ Diphyodonty: two sets of teeth, a juvenile dentition that is replaced by an adult set (e.g., many mammals, including humans). (E) Polyphyodonty: multiple rows of teeth (or patches) with teeth being shed and new teeth developing from adjacent loci to replace them throughout life (e.g., most fishes, amphibians, and reptiles). (F) Polystichy: multiple rows of teeth with several rows of functional teeth accumulated on the jaws (or in patches) without being serially shed (e.g., sharks). $(G)$ Edentulous: tooth development suppressed (e.g., some frogs, birds). Note that $A, B, C$, and $G$ may not involve replacement, depending on the lineage. Functional teeth are blue; replacement teeth in loci are white. 
I.K. Hariharan et al.

their relatives lose the teeth in their mouths and on their upper pharyngeal elements 50 million years ago? Regional loss of expression of the ectodysplasin-A (Eda) signaling ligand appears to be the likely cause of dentition reduction; overexpression of the gene in zebrafish restores teeth to the upper pharynx. Because teeth are not restored to the mouth, although competent to respond to Eda, the constraint appears to be the irreversible alteration of multiple genetic pathways required for tooth development. Consequently, a commonality of both constraint and selection likely is involved in tooth loss (Aigler et al. 2014). Conversely, Eda is implicated in many phases of tooth development and evolution, as well as tooth loss. Ingenious manipulation of Eda levels in explants and in Edanull mice has revealed the developmental and evolutionary patterns of development of the cusps and their shapes and numbers in mammals (Harjunmaa et al. 2014)

Several factors interact to restrict indeterminate tooth replacement in amniotes: loss of integrity of the dental lamina and loss of dental epithelial stem cells, inability to reform a successional lamina, and/or partial development of a nonfunctional successional lamina. The existence of an inhibitory mechanism similar to zones of inhibition has been predicted (Whitlock and Richman 2013). Several genes appear to be associated with tooth spacing and replacement, based on review of genetic/developmental mechanisms involved in changes in tooth complexity and replacement (fishes, reptiles, and mammals) (Jernvall and Thesleff 2012). Mammals have the most complex teeth among vertebrates, but have restricted capacity for renewal and replacement, because new tooth knots do not develop as in animals with polyphyodont teeth. Loss of tooth replacement has been compensated for by continuous growth of teeth in some mammals (e.g., incisors in some mice, rats, and rabbits, and molars in voles, some rabbits, and ferrets) (Jernvall and Thesleff 2012), although the evolutionary/functional basis for that modification is not known. The genetic basis for continuous growth and the gene expression patterns and the nature of the topography of mammalian teeth as they develop and evolve have been quantified (Jernvall et al. 2000). Jernvall's gene network model for development and evolution of mammalian teeth leads to several predictions (Jernvall 2002). The developmental pattern that amplifies the complexity of teeth apparently also restricts replacement, and it appears to "reuse" the regulatory pathways of tooth replacement. The specificity of enamel deposition apically, and growth basally, is a topic of intensive research; the molecular cascades that mediate shape change and growth and replacement patterns are thought to mediate the evolutionary and intraindividual modifications, but the triggers for different growth and replacement processes are not yet known (Jernvall and Thesleff 2012).

\section{Tooth Replacement}

Is tooth replacement simply an extended form of growth following tooth initiation? That is, specifically, are the genes involved in patterning tooth replacement the same as those that initiate primary teeth? For example, in the rainbow trout, three genes, all essential for mouse tooth development, have identical spatial-temporal expression in both taxa. At all tooth bud sites, epithelial genes initiate the morphogenetic cascade, effecting Bmp-4 expression in dental mesenchyme, including the formation of replacement teeth. The sequence is the same for oral jaw teeth, but one of the epithelial genes is deployed differently in the pharyngeal dentition. Several genes are operational in tooth development in both fishes and mammals, and may represent a stable vertebrate pathway because the osteichthyan divergence occurred 420 million years ago (Fraser et al. 2006). Furthermore, assessment of gene expression patterns in goldfish of a subset of genes involved in tooth initiation in mouse showed signaling differences in position and timing of the "mouse" genes at initiation and in replacement in goldfish. These genes may mark the sites of "set-aside cells," which give rise to further generations of teeth. This phenomenon may illustrate translation of initial pattern formation to secondary tooth-replacement patterns (Fraser et al. 2004). Related gene sets are involved in 
the differentiation of replacement teeth that have different shapes in Lake Malawi cichlid fishes, and there are dose-dependent effects on both replacement and tooth shape. Both tooth replacement and tooth-shape development appear to be integrated through a common set of molecular signals; integration has been lost or decoupled in the evolution of mammalian tooth development (Fraser et al. 2013).

Initiation of tooth replacement has several genetic causes. Huysseune's data for zebrafish (Huysseune 2006) support a local control mechanism of signaling in the dental lamina that initiates the formation of replacement teeth. Furthermore, in some snakes and lizards, Shh is necessary for tooth morphogenesis, but Shh is not involved in successional dental lamina formation, indicating that other pathways (as yet undetermined) regulate tooth replacement. Wnt read-out genes were expressed persistently, such that the molecular signaling mechanism carries over from tooth initiation to tooth replacement (Handrigan and Richman 2010a,b). These disparate data show the necessity of studying diverse taxa and of cross-comparing the data before inferring general patterns and processes. The full array of signaling systems and their organization in tooth development and replacement has yet to be elucidated.

The maintenance of tooth position and shape during ontogeny has been studied in several taxa. In alligators, which have simple, conical, socketed, continuously replaced teeth, but with morphologically distinct tooth types, there is significant morphological and genetic variation in early dental fates. Developmental variation results from a combination of modularity and semi-autonomous tooth development. Modularity may have been key to the adaptive evolution of teeth in amniotes, permitting progressive modification in tooth shape, number, and replacement pattern, and the alligator may be a model for the "most primitive" extant tetrapod dentition (Weeks et al. 2013).

\section{Stem Cells in Tooth Development}

An attractive notion is that the ability to replace teeth or to keep teeth in a continuously growing state requires the persistence of stem-cell populations. Indeed, stem cells and their involvement in tooth development have been studied in a diversity of vertebrate taxa. In some lizards, the formation of the dental lamina and production of its changes, including tooth replacement, are now thought to involve sequestered stem cells. In one experiment, cells that express genes that are markers of adult stem cells in mammals were found in pockets close to the successional dental lamina. Perhaps they represent a stemcell population whose descendants form the successional lamina and then the replacement teeth, and these dental stem cells are sequestered from signals that might otherwise induce them to differentiate earlier (Handrigan et al. 2010; Richman and Handrigan 2011). An epithelial stem-cell niche also is present in zebrafish and other fish (Huysseune and Thesleff 2004).

Many similarities are reported for the role of stem cells in hair growth and tooth replacement, including their sequestration of stem cells in bulbs in hair and knots in teeth (Huysseune and Thesleff 2004). Hair and tooth development may be regulated by the same molecular cascades, based on studies of human malformation syndromes and their occurrence in mouse mutant models. Similar molecular cascades underlie both hair and tooth cycling, so stem cells likely have a role in the formation of tooth buds for continuous tooth renewal. Furthermore, the putative stem cells have been identified in mouse and rat incisors and rabbit and vole molars, all nonreplacing teeth that have continuous growth. Huysseune and Thesleff note that FGF10 likely regulates the fate of determination of epithelial stem cells in many vertebrates. Despite the differences in structure of the enamel organ in most bony fish from that of mammals, the presence of sequestered stem cells and the molecular cascades regulating their involvement in tooth development, replacement, and growth warrants further investigation.

Studies of tooth development and replacement are providing information about many questions relevant to major questions about growth. Such issues as the bases of regulation of growth during ontogenies (including postmaturation), and the evolution of patterns of 
I.K. Hariharan et al.

growth, including similarities, differences, and phylogenetic correlations are being illuminated. Likewise, differences and similarities of initiation and replacement, and the roles of stem cells and cell cycles, as well as the genetic properties of local and whole-organism growth are under study. However, the paucity of comparable comparative data precludes any synthesis that might resolve major questions. No studies are available that elucidate the genetics of the entire sequential pattern of tooth development and replacement. Such research has the potential to provide information about the onset and offset signals that regulate the properties of ontogenies. Tooth development and its association of genes, signaling pathways, molecular cascades, and regulation have the potential to provide a paradigm for understanding the mechanisms of processes of growth and their modifications through the course of vertebrate evolution.

\section{CONCLUDING REMARKS}

When we first set out to write this review, we had assumed that most organisms arrest their growth at a size that is determined by their genetic program and that there are occasional examples of organisms that have devised ways of evading this constraint. Growth, even in these organisms, appears to slow down with age possibly because of constraints on organismal physiology raising the possibility that they simply die before they reach a final size. However, after examining the issue of growth termination in diverse taxa, we now favor the view that the ancestral condition is one of indeterminate growth and that determinate growth is a derived state. All multicellular organisms are derived from unicellular ancestors whose clonal progeny could keep multiplying indefinitely. This type of growth is likely to have been inherited by the earliest multicellular organisms. Simple organisms, such as placozoans, clearly lack a growthtermination program and continue to grow indefinitely but with periodic partition of the original organism into smaller progeny. If indeed indeterminate growth is the ancestral condition, then, as we have discussed herein, the definitive termination of growth seems to have occurred in different taxa in mechanistically distinct ways. Another important principle that emerges is that different organs within the same organism can differ with respect to whether they achieve a truly determinate state of growth. An understanding the precise mechanisms by which a pattern of indeterminate growth is modified in a variety of taxa to different types of determinate growth might be most tractable in a specific tissue, such as a comparative study of tooth development.

Interestingly, many of the organisms that are capable of tissue regeneration seem to be those that do not seem to have truly reached the end of their growth phase. For example, oligochaete worms remain capable of posterior regenerations, whereas leeches cannot. Cockroaches can regenerate limbs but only before their final molt. Salamanders, such as the axolotls, have prodigious abilities to regenerate parts, such as limbs and tails, and even parts of the brain. And planarians, which display remarkable plasticity in terms of body size, may be the champions of regeneration. Thus, the ability of homologous structures to regenerate in one species but not in another could reflect the extent to which growth is effectively terminated in those two species rather than the acquisition or loss of a separate regeneration program. If this view is correct, then no special genetic mechanisms may be required for regeneration; indeed, regeneration could potentially be viewed as a resumption of "normal" growth following a perturbation. This correlation between the termination of growth and the loss of regenerative capacity raises the intriguing possibility that regeneration is a "side benefit" of a growth program that has not been fully terminated and that it may not be adaptive in itself. Although regeneration can occur by diverse mechanisms (Tanaka and Reddien 2011), the similarities between regenerative growth and normal development within an individual species can often exceed similarities in regenerative growth among diverse species.

Finally, while researching the material presented here, we were struck by how little is known about the differences in growth-regula- 
tory mechanisms between related taxa that show determinate and indeterminate growth. In addition to a careful characterization of the underlying differences at the cellular level, the combination of genome sequencing with recent developments in genome-editing technologies should enable a deeper understanding of the mechanisms that arrest growth in a wide variety of animals.

\section{ACKNOWLEDGMENTS}

M.H.W. thanks students and colleagues for many discussions of the evolution and development of patterns and processes of growth, especially of teeth, over the years, Jukka Jernvall for his rare insight and integrative approach to the biology of teeth, and the National Science Foundation for its many years of support of her research program. I.K.H. thanks David Weisblat, Nipam Patel, Rebecca Heald, Alejandro Sánchez-Alvarado, and Jaume Baguña for advice, discussions, and/or comments on the manuscript. I.K.H. is funded by the National Institutes of Health (NIH) and an American Cancer Society Research Professor Award (120366-RP-11-078-01-DDC).

\section{REFERENCES}

Abeloos M. 1930. Recherches expérimentales sur la croissance et la régénération chez les Planaires [Experimental research on development and regeneration in planarians]. Bull Biol France Belg 64: 1-140.

Aigler SR, Jandzik D, Hatta K, Uesugi K, Stock DW. 2014. Selection and constraint underlie irreversibility of tooth loss in cypriniform fishes. Proc Natl Acad Sci 111: 77077712

Alberch P. 1980. Ontogenesis and morphological diversification. Am Zool 20: 653-667.

Alberch P, Gould SJ, Oster GF, Wake DB. 1979. Size and shape in ontogeny and phylogeny. Paleobiology 5: 296-317.

Baguña J. 1998. Planarians. In Cellular and molecular basis of regeneration (ed. Ferretti P, Géraudie J), pp. 135-165. Wiley, Chichester, UK.

Baguña J, Romero R. 1981. Quantitative analysis of cell types during growth, degrowth and regeneration in the planarians Dugesia mediterranea and Dugesia tigrina. Hydrobiologia 84: 181-194.

Barry ER, Morikawa T, Butler BL, Shrestha K, de La Rosa R, Yan KS, Fuchs CS, Magness ST, Smits R, Ogino S. 2013. Restriction of intestinal stem cell expansion and the regenerative response by YAP. Nature 493: 106-110.
Bely AE, Sikes JM. 2010. Latent regeneration abilities persist following recent evolutionary loss in asexual annelids. Proc Natl Acad Sci 107: 1464-1469.

Bodenstein D. 1953. Studies on the humoral mechanisms in growth and metamorphosis of the cockroach, Periplaneta americana. II: The function of the prothoracic gland and the corpus cardiacum. J Exp Zool 123: 413-433.

Buttitta LA, Edgar BA. 2007. Mechanisms controlling cell cycle exit upon terminal differentiation. Curr Opin Cell Biol 19: 697-704.

Camargo FD, Gokhale S, Johnnidis JB, Fu D, Bell GW, Jaenisch R, Brummelkamp TR. 2007. YAP1 increases organ size and expands undifferentiated progenitor cells. Curr Biol 17: 2054-2060.

Dai JD, Gilbert LI. 1991. Metamorphosis of the corpus allatum and degeneration of the prothoracic glands during the larval-pupal-adult transformation of Drosophila melanogaster: A cytophysiological analysis of the ring gland. Dev Biol 144: 309-326.

de Rosa R, Prud'homme B, Balavoine G. 2005. caudal and even-skipped in the annelid Platynereis dumerilii and the ancestry of posterior growth. Evol Dev 7: 574-587.

Dong J, Feldmann G, Huang J, Wu S, Zhang N, Comerford SA, Gayyed MF, Anders RA, Maitra A, Pan D. 2007. Elucidation of a universal size-control mechanism in Drosophila and mammals. Cell 130: 1120-1133.

Fabrezi M, Quinzio S, Goldberg J. 2009. Giant tadpole and delayed metamorphosis of Pseudis platensis Gallardo, 1961 (Anura, Hylidae). J Herpetol 43: 228-243.

Ferrier DE. 2012. Evolutionary crossroads in developmental biology: Annelids. Development 139: 2643-2653.

Fischer AH, Henrich T, Arendt D. 2010. The normal development of Platynereis dumerilii (Nereididae, Annelida). Front Zool 7: 31.

Fraser GJ, Graham A, Smith MM. 2004. Conserved deployment of genes during odontogenesis across osteichthyans. Proc Biol Sci 271: 2311-2317.

Fraser GJ, Berkovitz BK, Graham A, Smith MM. 2006. Gene deployment for tooth replacement in the rainbow trout (Oncorhynchus mykiss): A developmental model for evolution of the osteichthyan dentition. Evol Dev 8: 446457.

Fraser GJ, Bloomquist RF, Streelman JT. 2013. Common developmental pathways link tooth shape to regeneration. Dev Biol 377: 399-414.

Gazave E, Béhague J, Laplane L, Guillou A, Préau L, Demilly A, Balavoine G, Vervoort M. 2013. Posterior elongation in the annelid Platynereis dumerilii involves stem cells molecularly related to primordial germ cells. Dev Biol 382: 246-267.

Giribet G, Edgecombe GD. 2012. Reevaluating the arthropod tree of life. Annu Rev Entomol 57: 167-186.

Gould SJ. 1977. Ontogeny and phylogeny. Harvard University Press, Boston.

Hadorn E. 1968. Transdetermination in cells. Sci Am 219: 110-120.

Handrigan GR, Richman JM. 2010a. Autocrine and paracrine Shh signaling are necessary for tooth morphogenesis, but not tooth replacement in snakes and lizards (Squamata). Dev Biol 337: 171-186. 
I.K. Hariharan et al.

Handrigan GR, Richman JM. 2010b. A network of Wnt, hedgehog and BMP signaling pathways regulates tooth replacement in snakes. Dev Biol 348: 130-141.

Handrigan GR, Leung KJ, Richman JM. 2010. Identification of putative dental epithelial stem cells in a lizard with lifelong tooth replacement. Development 137: 3545-3549.

Hanken J. 1982. Appendicular skeletal morphology in minute salamanders, genus Thorius (Amphibia: Plethodontidae): Growth regulation, adult size determination, and natural variation. J Morphol 174: 57-77.

Hanken J, Wake DB. 1992. Miniaturization of body size: Organismal consequences and evolutionary significance. Annu Rev Ecol Syst 24: 501-519.

Harjunmaa E, Seidel K, Hakkinen T, Renvoise E, Corfe IJ, Kallonen A, Zhang ZQ, Evans AR, Mikkola ML, SalazarCiudad I, et al. 2014. Replaying evolutionary transitions from the dental fossil record. Nature 512: 44-48.

Hartnoll R. 1983. Strategies of crustacean growth. Aust Mus Syd Mem 18: 121-131.

Hartnoll RG. 2001. Growth in Crustacea-Twenty years on. In Advances in decapod Crustacean research, pp. 111-122. Kluwer Academic, Norwell, MA.

Huxley JS. 1932. Problems of relative growth. Methuen, London.

Huysseune A. 2006. Formation of a successional dental lamina in the zebrafish (Danio rerio): Support for a local control of replacement tooth initiation. Int J Dev Biol 50: $637-643$.

Huysseune A, Thesleff I. 2004. Continuous tooth replacement: The possible involvement of epithelial stem cells. BioEssays 26: 665-671.

Jernvall J. 2002. A gene network model accounting for development and evolution of mammalian teeth. Proc Natl Acad Sci 99: 8116-8120.

Jernvall J, Thesleff I. 2012. Tooth shape formation and tooth renewal: Evolving with the same signals. Development 139: 3487-3497.

Jernvall J, Keränen SV, Thesleff I. 2000. Evolutionary modification of development in mammalian teeth: Quantifying gene expression patterns and topography. Proc Nat Acad Sci 97: 14444-14448.

Lachaise F, Le Roux A, Hubert M, Lafont R. 1993. The molting gland of crustaceans: Localization, activity, and endocrine control (A review). J Crustacean Biol 13: 198-234.

Lin AY, Pearson BJ. 2014. Planarian yorkie/YAP functions to integrate adult stem cell proliferation, organ homeostasis and maintenance of axial patterning. Development 141: 1197-1208.

Liu N-J, Isaksen DE, Smith C, Weisblat D. 1998. Movements and stepwise fusion of endodermal precursor cells in leech. Dev Genes Evol 208: 117-127.

Mané-Padrós D, Cruz J, Vilaplana L, Pascual N, Bellés X, Martín D. 2008. The nuclear hormone receptor BgE75 links molting and developmental progression in the direct-developing insect Blattella germanica. Dev Biol 315: $147-160$.

Mané-Padrós D, Cruz J, Vilaplana L, Nieva C, Ureña E, Bellés X, Martín D. 2010. The hormonal pathway controlling cell death during metamorphosis in a hemimetabolous insect. Dev Biol 346: 150-160.
McNabb FMA, Scanes CG, Zeman M. 1998. Endocrine control of development. In Avian growth and development (ed. Starck JM, Ricklefs RE). Oxford University Press, New York.

Miller CM, Newmark PA. 2012. An insulin-like peptide regulates size and adult stem cells in planarians. Int J Dev Biol 56: $75-82$.

Müller HC. 1913. Die Regeneration der Gonophore bei den Hydroiden und anschliessende Biologische Beobachtungen. I: Athecata [The regeneration of the gonophore in hydroids and subsequent biological observations]. Entwicklungsmech Org 37: 319-419.

Newmark PA, Reddien PW, Cebria F, Sanchez Alvarado A. 2003. Ingestion of bacterially expressed double-stranded RNA inhibits gene expression in planarians. Proc Natl Acad Sci 100: 11861-11865.

Nijhout HF, Riddiford LM, Mirth C, Shingleton AW, Suzuki Y, Callier V. 2014. The developmental control of size in insects. Wiley Interdiscip Rev Dev Biol 3: $113-134$.

Oviedo NJ, Newmark PA, Sanchez Alvarado A. 2003. Allometric scaling and proportion regulation in the freshwater planarian Schmidtea mediterranea. Dev Dyn 226: 326-333.

Piraino S, De Vito D, Schmich J, Bouillon J, Boero F. 2004. Reverse development in Cnidaria. Can J Zool 82: $1748-$ 1754.

Pucek Z. 1957. Histomorphologische Untersuchungen über die Winterdepression des Schädels bei Sorex L. und Neomys Kaup [Histomorphological investigation of the winter depression of the skull of Sorex L. and Neomys Kaup]. Ann Univ Mariae Curie-Sklodowska Lublin Poland 10: 399-425.

Rewitz KF, Yamanaka N, O'Connor MB. 2013. Developmental checkpoints and feedback circuits time insect maturation. Curr Top Dev Biol 103: 1-33.

Richards OW, Davies RG. 1977. Thysanura (Bristle-Tails; Silverfish). In IMMS' general textbook of entomology, pp. 433-443. Chapman \& Hall, London.

Richman JM, Handrigan GR. 2011. Reptilian tooth development. Genesis 49: 247-260.

Schmich J, Kraus Y, De Vito D, Graziussi D, Boero F, Piraino S. 2007. Induction of reverse development in two marine hydrozoans. Int J Dev Biol 51: 45.

Sebens KP. 1987. The ecology of indeterminate growth in animals. Annu Rev Ecol System 18: 371-407.

Secor SM, Diamond J. 1997. Effects of meal size on postprandial responses in juvenile Burmese pythons (Python molurus). Am J Phys 41: R902.

Shine R, Charnov EL. 1992. Patterns of survival, growth, and maturation in snakes and lizards. Am Nat 139: $1257-$ 1269.

Swanson EM, McElhinny TL, Dworkin I, Wedele ML, Glickman SE, Holekamp KE. 2013. Ontogeny of sexual size dimorphism in the spotted hyena (Crocuta crocuta). $J$ Mammal 94: 1298-1310.

Tanaka EM, Reddien PW. 2011. The cellular basis for animal regeneration. Dev Cell 21: 172-185.

Wainwright TC, Armstrong DA. 1993. Growth patterns in the Dungeness crab (Cancer magister Dana): Synthesis 
Indeterminate Growth

of data and comparison of models. J Crustacean Biol 13: 36-50.

Webster S, Keller R. 1986. Purification, characterisation and amino acid composition of the putative moult-inhibiting hormone (MIH) of Carcinus maenas (Crustacea, Decapoda). J Comp Physiol B 156: 617-624.

Weeks O, Bhullar BAS, Abzhanov A. 2013. Molecular characterization of dental development in a toothed archosaur, the American alligator Alligator mississippiensis. Evol Dev 15: 393-405.
Whitlock JA, Richman JM. 2013. Biology of tooth replacement in amniotes. Int J Oral Sci 5: 66-70.

Wikelski M, Thom C. 2000. Marine iguanas shrink to survive El Niño. Nature 403: 37-38.

Yampolsky LY, Ebert D. 1994. Variation and plasticity of biomass allocation in Daphnia. Funct Ecol 8: 435-440.

Zhou S, Shirley TC, Kruse GH. 1998. Feeding and growth of the red king crab Paralithodes camtschaticus under laboratory conditions. J Crustacean Biol 18: $337-345$. 


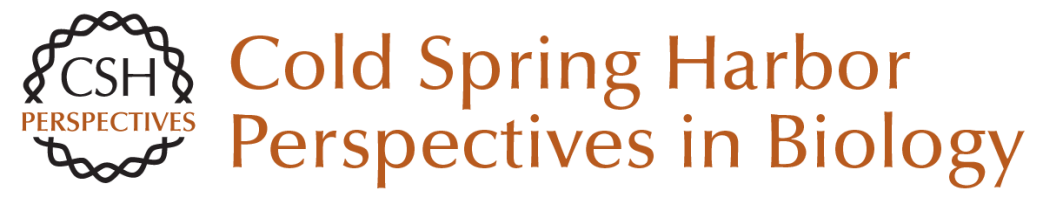

\section{Indeterminate Growth: Could It Represent the Ancestral Condition?}

Iswar K. Hariharan, David B. Wake and Marvalee H. Wake

Cold Spring Harb Perspect Biol 2016; doi: 10.1101/cshperspect.a019174 originally published online July 27, 2015

\section{Subject Collection Size Control in Biology: From Organelles to Organisms}

Cell-Size Control

Amanda A. Amodeo and Jan M. Skotheim

Indeterminate Growth: Could It Represent the

Ancestral Condition?

Iswar K. Hariharan, David B. Wake and Marvalee H. Wake

The Systemic Control of Growth Laura Boulan, Marco Milán and Pierre Léopold

Genome Biology and the Evolution of Cell-Size Diversity

Rachel Lockridge Mueller

Size Scaling of Microtubule Assemblies in Early Xenopus Embryos

Timothy J. Mitchison, Keisuke Ishihara, Phuong Nguyen, et al.

The Influence of Genome and Cell Size on Brain Morphology in Amphibians Gerhard Roth and Wolfgang Walkowiak

The Opposing Actions of Target of Rapamycin and AMP-Activated Protein Kinase in Cell Growth Control

Sravanth K. Hindupur, Asier González and Michael N. Hall

Small but Mighty: Cell Size and Bacteria

Petra Anne Levin and Esther R. Angert
Mechanical Forces and Growth in Animal Tissues Loïc LeGoff and Thomas Lecuit

Biological Scaling Problems and Solutions in

Amphibians

Daniel L. Levy and Rebecca Heald

Intracellular Scaling Mechanisms Simone Reber and Nathan W. Goehring

Growing an Embryo from a Single Cell: A Hurdle in Animal Life Patrick H. O'Farrell

Organ-Size Regulation in Mammals Alfredo I. Penzo-Méndez and Ben Z. Stanger

Size Control in Plants--Lessons from Leaves and Flowers

Hjördis Czesnick and Michael Lenhard

Nuclear DNA Content Varies with Cell Size across

Human Cell Types

James F. Gillooly, Andrew Hein and Rachel

Damiani

Subcellular Size

Wallace F. Marshall

For additional articles in this collection, see http://cshperspectives.cshlp.org/cgi/collection/

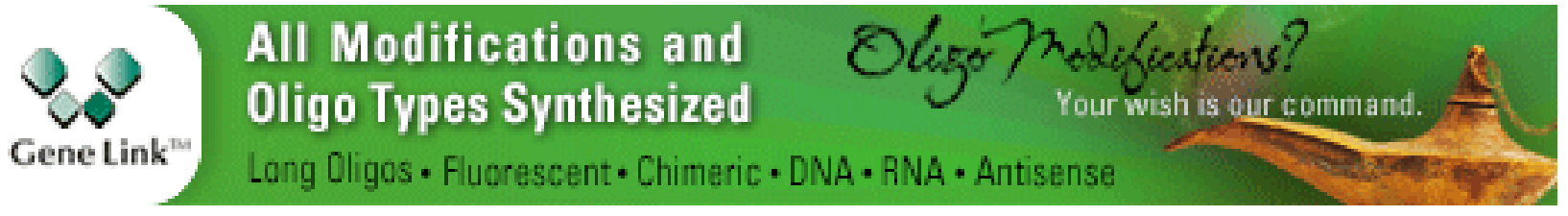

Copyright @ 2016 Cold Spring Harbor Laboratory Press; all rights reserved 